J Urol. 2007 December ; 178(6): 2359-2365. doi:10.1016/j.juro.2007.08.039.

\title{
Expectant Management of Prostate Cancer With Curative Intent: An Update of The Johns Hopkins Experience
}

\author{
H. Ballentine Carter ${ }^{*}$, Anna Kettermann, Christopher Warlick, E. Jeffrey Metter, Patricia \\ Landis, Patrick C. Walsh, and Jonathan I. Epstein \\ Departments of Urology (CW, AK, PL, PCW, JIE, HBC) and Pathology (JIE), The Johns Hopkins \\ University School of Medicine and The James Buchanan Brady Urological Institute, The Johns \\ Hopkins Hospital and National Institute on Aging, National Institutes of Health, Clinical Research \\ Branch (EJM, AK), Baltimore, Maryland
}

\begin{abstract}
Purpose-We updated our experience with a strategy of expectant treatment for men with stage T1c prostate cancer and evaluated predictors of disease intervention.

Materials and Methods-A total of 407 men with a median age of 65.7 years (range 45.8 to $81.5)$ with stage T1c $(99.8 \%)$ or T2a $(0.2 \%)$ prostate cancer suspected of harboring small volume prostate cancer based on needle biopsy findings and prostate specific antigen density have been followed in a prospective, longitudinal surveillance program with a median followup of 2.8 years (range 0.4 to 12.5). A recommendation for treatment was made if disease progression was suggested by unfavorable followup needle biopsy findings (Gleason pattern 4 or 5, greater than 2 biopsy cores with cancer or greater than 50\% involvement of any core with cancer). Cox proportional hazards regression was used to evaluate the affect of multiple covariates on the outcome of curative intervention.
\end{abstract}

Results-Of 407 men 239 (59\%) men remained on active surveillance at a median followup of 3.4 years (range 0.43 to 12.5$), 103$ (25\%) underwent curative intervention at a median of 2.2 years after diagnosis (range 0.96 to 7.39 ) and 65 (16\%) were either lost to followup (12), withdrew from the program (45), or died of causes other than prostate cancer (8). Older age at diagnosis ( $\mathrm{p}=$ $0.011)$ and an earlier date of diagnosis $(p=0.001)$ were significantly associated with curative intervention.

Conclusions-Recognizing that over treatment of prostate cancer is prevalent, especially among elderly patients, a program of careful selection and monitoring of older men who are likely to harbor small volume, low grade disease may be a rational alternative to the active treatment of all.

\section{Keywords}

prostate; prostatic neoplasms; follow-up studies; age factors

\footnotetext{
Copyright $@ 2007$ by American Urological Association

* Correspondence: Department of Urology, 145 Marburg Building, Johns Hopkins Hospital, 600 North Wolfe St., Baltimore, Maryland 21287-2101 (telephone: 410-955-0351; FAX: 410-955-0833; hcarter@jhmi.edu)..

Study received approval from the institutional review board at The Johns Hopkins Medical Institution.
} 
The widespread use of PSA as a biomarker of prostate cancer and the ease of sampling prostate tissue with transrectal ultrasound directed prostate biopsy, combined with the high prevalence of biologically indolent disease, have resulted in the detection and treatment of many cancers that would have remained undetected during life in the absence of screening. ${ }^{1}$ Most men diagnosed with prostate cancer today have low to intermediate risk prostate cancers diagnosed at a median age of 68 years and more than $90 \%$ of them undergo active treatments. ${ }^{2}$ Overtreatment of prostate cancer is more common among older men. The absolute decrease in prostate cancer specific death in men 65 years or older randomized to surgery vs observation was $0.3 \%$ at 10 years, compared to $11 \%$ in those younger than 65 years. ${ }^{3}$ Based on this trial of nonscreen detected cancers, more than 300 men 65 years or older would need to undergo surgery to prevent 1 prostate cancer death at 10 years. Thus, it seems evident that the majority of older men diagnosed today with screen detected prostate cancer do not gain years of life with curative intervention.

An alternative to the curative intervention of all men with a cancer diagnosis is the careful selection and monitoring of older men considered to have low risk disease, with the intent to cure should the disease progress. Since 1995, we have enrolled men into a program of expectant management with curative intent ${ }^{4}$ and we update our experience with this approach.

\section{METHODS}

\section{Cohort Enrollment}

Since 1995, older men seeking consultation at our institution with suspected small, lower grade prostate cancer have been counseled, at the discretion of the physician, that expectant management with curative intent is an acceptable alternative to curative intervention. ${ }^{4}$ Younger men have chosen expectant management because of personal reasons or comorbidity. This prospective longitudinal program has been approved by the institutional review board at The Johns Hopkins Medical Institution and all men signed an informed consent prior to entering the program. Approximately $1 \%$ to $2 \%$ of the patients with newly diagnosed prostate seen at our institution have entered this program. During the last year, $46 \%$ of patients who were considered candidates for expectant management and received written materials on the program entered the surveillance program at our institution.

Enrollment criteria were originally described by Epstein et al. ${ }^{5}$ The criteria used in this program are based on PSAD (PSA before diagnosis divided by prostate volume determined by transrectal ultrasound measurement) and needle biopsy findings in patients with nonpalpable prostate cancers (stage T1c), including PSAD $0.15 \mathrm{ng} / \mathrm{ml} / \mathrm{cm}^{3}$ or less and favorable biopsy characteristics (Gleason score 6 or less with no Gleason pattern grade of 4 or 5, no more than 2 cores positive for cancer, and no more than $50 \%$ of any 1 core involved with cancer).

\section{Plan for Surveillance}

Followup of men treated expectantly was semiannual measurements of total and free PSA, a semiannual digital rectal examination, and an annual surveillance prostate biopsy 
examination. The mean $\pm \mathrm{SD}$ time from the diagnostic biopsy to the first annual surveillance biopsy was $0.85 \pm 0.54$ years. All men in this program agreed to undergo surveil-lance biopsies and the mean time between biopsies was $1.0 \pm 0.49$ year. Curative intervention (radiation or surgery) was triggered by the finding of adverse pathological features on an annual surveillance biopsy examination (a Gleason score of 7 or greater, or a Gleason pattern grade 4 or 5 , more than 2 cores that were positive for cancer, or more than $50 \%$ of any 1 core that was involved with cancer) or a patient request for a change in management. More recently we have encouraged men with the finding of perineural invasion on biopsy to undergo curative intervention because of the possibility that this finding could place them at higher risk for biologically significant disease. Thus, in our program, PSA changes did not trigger intervention.

\section{Statistical Analysis}

Study group characteristics at diagnosis (age, PSA, PSAD, the number of cores positive for cancer and the maximum percent core involvement with cancer), year of diagnosis, percent free PSA within a year of diagnosis, and PSA kinetics (velocity and doubling time), based on all PSA values after diagnosis, were compared using the pooled $t$ test in the case of equal variances and the Cochran $t$ test in the case of unequal variances with $p<0.05$ considered statistically significant.

Time to curative intervention (the event) was estimated using the Kaplan-Meier method with censoring of subjects who were active as of November 2006. Time 0 was defined as the time at diagnosis, and followup time was defined as time from diagnosis to the date of curative intervention for subjects who underwent treatment, or November 2006 for subjects who remained active in the program. Censoring for subjects lost to followup and who withdrew from the program was at the date of the last visit, and for those deceased it was at the time of death.

PSAV was calculated using linear regression analysis of all PSA measurements during the time of followup after diagnosis. PSADT was calculated as the natural log of $2(0.693)$ divided by the slope of the linear regression line of log of PSA with time using all PSA values available after the diagnosis. Cox proportional hazards regression was used to evaluate the effect of covariates on the main outcome, that is curative intervention.

\section{RESULTS}

As of November 7, 2006, there were 407 men enrolled in the expectant management program. Table 1 shows the enrollment characteristics of this cohort. While most men met the enrollment PSAD criteria (83\%) and the biopsy criteria (98\%), 19\% overall (77) did not meet these criteria. Of these 77 men, 38 (49\%) remained active in the program for personal reasons, eg the desire to avoid treatment, or due to comorbidities, and primarily because PSAD slightly higher than $0.15 \mathrm{ng} / \mathrm{ml} / \mathrm{cm}^{3}$ was the only adverse factor. Of those subjects who met the PSAD and the biopsy criteria (330), 23 (7\%) chose to undergo treatment after enrollment in the program, although there were no adverse findings at followup. 
Of 407 men in the expectant management program, 239 (59\%) were active, $103(25 \%)$ underwent curative intervention, $45(11 \%)$ had withdrawn from the program, $12(3 \%)$ were lost to followup, and 8 (2\%) had died of causes other than prostate cancer.

An estimate of freedom from curative intervention showed that the median time to intervention had not been attained, but few men have been followed beyond 5 to 6 years (fig. 1). A steeper decrease in freedom from intervention in the earliest years could represent initial under sampling of cancer on biopsy during a time when the routine biopsy approach was 6 cores instead of 12 or more. For each year prior to and including 1999, 27\% or more of the subjects diagnosed were treated within 3 years of diagnosis. By comparison, for each year after 1999, 16\% or fewer subjects were treated within 3 years of diagnosis. These data suggest that under sampling of cancer was more common prior to 1999 , leading to a followup surveillance biopsy that was more likely to identify unfavorable disease.

Of 103 men who underwent curative intervention, 53 (51\%) underwent radical prostatectomy. Based on a prior definition of noncurable disease, that is stage pT2 organ confined if Gleason sum was 7 or greater $(4+3)$ and/or the surgical margins were positive any grade, stage pT3aN0 (extraprostatic extension) if Gleason sum was 7 or greater and/or surgical margins were positive, any stage higher than pT3a regardless of grade or margin status or any $\mathrm{N}+$ stage,${ }^{6} 20 \%$ of the men (10 of 49$)$ for whom pathological findings were available for review at our institution had noncurable disease. We previously reported a similar rate of noncurable disease among men in this program undergoing delayed surgical intervention that did not differ from that among men who met the inclusion criteria for the expectant management program but underwent immediate surgical intervention. ${ }^{6}$

PSA at diagnosis, PSAD at diagnosis, and year of diagnosis were statistically significantly different when comparing the treated and untreated groups for all subjects (table 2). Among the subset of subjects who met all program entry criteria, PSA at diagnosis, percent free PSA at diagnosis, PSAD at diagnosis, PSAV (slope), and year of diagnosis were statistically significantly different when comparing the treated and untreated groups. An earlier date of diagnosis may be associated with a greater risk of treatment because of a higher likelihood of under sampling of cancer in earlier years, as described, and/or because of stage migration that occurred with time in the PSA era. On multivariate analysis, older age at diagnosis and earlier date of diagnosis were significantly associated with the risk of curative intervention when considering all subjects (table 3). Among the subset of subjects who met all program entry criteria, age at diagnosis, PSAD at diagnosis, PSAV and year of diagnosis were significantly associated with the risk of curative intervention. PSAD and PSAV more closely correlated when considering all subjects compared to the subset of subjects who met all program entry criteria (Pearson correlation coefficient $r=0.27$ vs 0.12 ).

A comparison of age, PSADT, PSAV, PSAD, and percent free PSA among subjects who did and did not undergo treatment demonstrated the overlapping values between the groups (fig. 2). 


\section{DISCUSSION}

We have provided an update of an expectant management program (active surveillance) begun in 1995 that has enrolled 407 subjects using predefined selection criteria that were shown to be predictive of small volume, low grade cancer. ${ }^{5}$ In this program an older age at diagnosis and an earlier date of diagnosis were significantly associated with delayed curative intervention. It is possible that in our program older men were more likely to have had cancer under sampled at diagnosis and an earlier date of diagnosis may have been associated with a greater risk of under sampling on needle biopsy. This could explain the higher rates of intervention for older men and those diagnosed at an earlier date. Among those subjects who met all of our program entry criteria, in addition to age at diagnosis and year of diagnosis, PSAD and PSAV were significantly associated with curative intervention. However, the overlapping values of potential predictors of intervention would make it difficult to identify men on an individual basis who are at risk for later treatment (fig. 2). Thus, we believe that surveillance biopsies are an important component of followup monitoring.

Our approach for selecting and monitoring men differs from that described by others and could be considered more conservative. ${ }^{7-10}$ In the only other large, prospective surveillance program with predefined selection and monitoring criteria, and published outcomes, men with clinical stage T2b or less, Gleason score of 7 or less, and PSA $15 \mathrm{ng} / \mathrm{ml}$ or less were enrolled. ${ }^{8}$ In contrast, all but 1 man in the current study had stage T1c disease, all had a biopsy Gleason score of 6 or less, and $83 \%$ had a PSAD of $0.15 \mathrm{ng} / \mathrm{ml} / \mathrm{cm}^{3}$ or less (table 1). Furthermore, we used the results of an annual surveillance prostate biopsy to trigger curative intervention, not PSA kinetics or clinical findings, eg digital rectal examination progression.

PSA kinetics have been shown to be a predictor of curative intervention in surveillance programs. ${ }^{8,10-12}$ Studies that use PSADT as a criteria to define progression and differentially remove from surveillance those with more rapid increases in PSA (shorter PSADT), leaving those with stable or decreasing PSA values on surveillance, would be likely to show a relationship between PSA kinetics and intervention. We believe that by the time PSA kinetics and clinical signs, eg digital rectal examination changes, suggest progression, the window of curability may have closed.

Using a pathological definition of incurable prostate cancer that correlates with less than a $75 \%$ chance of remaining biochemically disease-free for 10 years, we previously reported that men in our surveillance program who underwent delayed surgical intervention had similar rates of incurable cancer compared to that in those who qualified for the surveillance program but underwent immediate surgical intervention. ${ }^{6}$ In our previous report the rate of incurable cancer for men undergoing delayed surgical intervention was $23 \%$ of 38 men, ${ }^{6}$ and in the current report it was $20 \%$ of the 49 for whom surgical pathological findings were reviewed at our institution. Recognizing that our definition of incurable disease is arbitrary and reflective of biochemical failure, not death from prostate cancer, these data suggest a low likelihood of losing the window of opportunity for cure in the short term when men are carefully selected and monitored. However, 2 men in our program were found to have lymph nodal involvement at surgery, 5 and 7 years, respectively, after entering the program. 
Both men had relatively high PSAD during followup but met all the biopsy criteria for remaining in the program. Because of this experience, we have decided to exclude men from the program if they fail to meet the PSAD criteria and have no serious comorbidity.

The majority of men diagnosed with prostate cancer today are older than 65 years, have low to intermediate risk cancer, and receive some form of active treatment. ${ }^{2}$ A substantial proportion of these men will gain no benefit from treatment in terms of additional years of life. In a randomized trial comparing surgically treated to untreated patients with prostate cancers that were not screen detected, more than 300 men 65 years or older would need to be treated to prevent 1 prostate cancer death during 10 years. ${ }^{3}$ These data are consistent with the 200 men older than 65 years who would need to undergo treatment to prevent 1 prostate cancer death during 12 years reported in an observational study of treatment vs observation. ${ }^{13}$ Thus, it is likely that the majority of men older than 65 years with screen detected, low risk prostate cancers undergo a treatment that does not lengthen life.

A number of limitations should be considered when interpreting our data. 1) Although prospective in nature, our study results are preliminary and our study design is not randomized, which limits our ability to determine the potential benefits or harm associated with surveillance. 2) Because our patients presented to a large tertiary referral center for advice regarding prostate cancer, and because we did not collect comorbidity indexes, our patient population may not be applicable to other patient populations in which the case mix could be different.

\section{CONCLUSIONS}

In summary, an alternative to the immediate treatment of all men with prostate cancer is the careful selection and monitoring of those suspected of harboring small volume, low grade disease with curative intent. We recognize that using the selection criteria that we described or that of others ${ }^{14,15}$ would result in the unnecessary treatment of some men, but hopefully minimize a missed opportunity for cure in the majority.

\section{Abbreviations and Acronyms}

PSA prostate specific antigen

PSAD PSA density

PSADT PSA doubling time

PSAV PSA velocity

\section{REFERENCES}

1. Draisma G, Boer R, Otto SJ, van der Cruijsen IW, Damhuis RA, Schroder FH, et al. Lead times and overdetection due to prostate specific antigen screening: estimates from the European Randomized Study of Screening for Prostate Cancer. J Natl Cancer Inst. 2003; 95:868. [PubMed: 12813170]

2. Cooperberg MR, Moul JW, Carroll PR. The changing face of prostate cancer. J Clin Oncol. 2005; 23:8146. [PubMed: 16278465] 
3. Holmberg L, Bill-Axelson A, Garmo H, Palmgren J, Norlén BJ, Adami HO, et al. Prognostic markers under watchful waiting and radical prostatectomy. Hematol Oncol Clin North Am. 2006; 20:845. [PubMed: 16861118]

4. Carter HB, Walsh PC, Landis P, Epstein JI. Expectant management of stage T1c prostate cancer with curative intent: preliminary results. J Urol. 2002; 167:1231. [PubMed: 11832703]

5. Epstein JI, Walsh PC, Carmichael M, Brendler CB. Pathologic and clinical findings to predict tumor extent of non-palpable (stage T1c) prostate cancer. JAMA. 1994; 271:368. [PubMed: 7506797]

6. Warlick C, Trock BJ, Landis P, Epstein JI, Carter HB. Pathological outcomes are similar for men in an expectant management program undergoing delayed surgical intervention compared to those undergoing immediate intervention. J Natl Cancer Inst. 2006; 98:355. [PubMed: 16507832]

7. Patel MI, DeConcini DT, Lopez-Corona E, Ohori M, Wheeler T, Scardino PT. An analysis of men with clinically localized prostate cancer who deferred definitive therapy. J Urol. 2004; 171:1520. [PubMed: 15017211]

8. Klotz L. Active surveillance for prostate cancer: for whom? J Clin Oncol. 2005; 23:8165. [PubMed: 16278468]

9. el-Geneidy M, Garzotto M, Panagiotou I, Hsieh YC, Mori M, Peters L, et al. Delayed therapy with curative intent in a contemporary prostate cancer watchful-waiting cohort. BJU Int. 2004; 93:510. [PubMed: 15008720]

10. Roemeling S, Roobol MJ, de Vries SH, Wolters T, Gosselaar C, van Leenders GJ, et al. Active surveillance for prostate cancers detected in three subsequent rounds of a screening trial: characteristics, PSA doubling times, and outcome. Eur Urol. 2007; 51:1244. [PubMed: 17161520]

11. Carter CA, Donahue T, Sun L, Wu H, McLeod DG, Amling C, et al. Temporarily deferred therapy (watchful waiting) for men younger than 70 years and with low-risk localized prostate cancer in the prostate specific antigen era. J Clin Oncol. 2003; 21:4001. [PubMed: 14581423]

12. Khatami A, Aus G, Damber JE, Lilja H, Lodding P, Hugosson J. PSA doubling time predicts the outcome after active surveillance in screening-detected prostate cancer: results from the European randomized study of screening for Prostate cancer, Sweden section. Int J Cancer. 2006; 120:170. [PubMed: 17013897]

13. Wong YN, Mitra N, Hudes G, Localio R, Schwartz SJ, Wan F, et al. Survival associated with treatment vs observation of localized prostate cancer in elderly men. JAMA. 2006; 296:2683. [PubMed: 17164454]

14. Kattan MW, Eastham JA, Wheeler TM, Maru N, Scardino PT, Erbersdobler A, et al. Counseling men with prostate cancer: a nomogram for predicting the presence of small, moderately differentiated, confined tumors. J Urol. 2003; 170:1792. [PubMed: 14532778]

15. Ochiai A, Trpkov K, Yilmaz A, Donnelly B, Babaian RJ. Validation of a prediction model for low volume/low grade cancer: application in selecting patients for active surveil-lance. J Urol. 2007; 177:907. [PubMed: 17296373] 


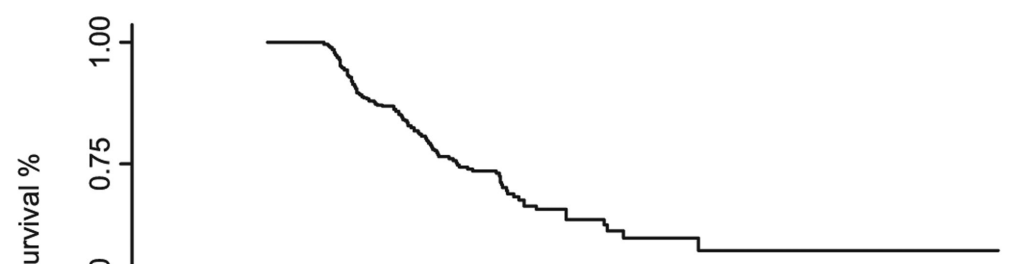

At risk:

\begin{tabular}{ccccccccccccc}
407 & \multicolumn{2}{c}{288} & \multicolumn{1}{c}{124} & & \multicolumn{2}{c}{42} & & 16 & & 8 & 1 \\
\hline 1 & 1 & 2 & 3 & 4 & 5 & 6 & 7 & 8 & 9 & 10 & 11 & 12
\end{tabular}

Time (yrs)

Fig. 1.

Kaplan-Meier estimate of treatment-free survival in 407 men in expectant management program. 

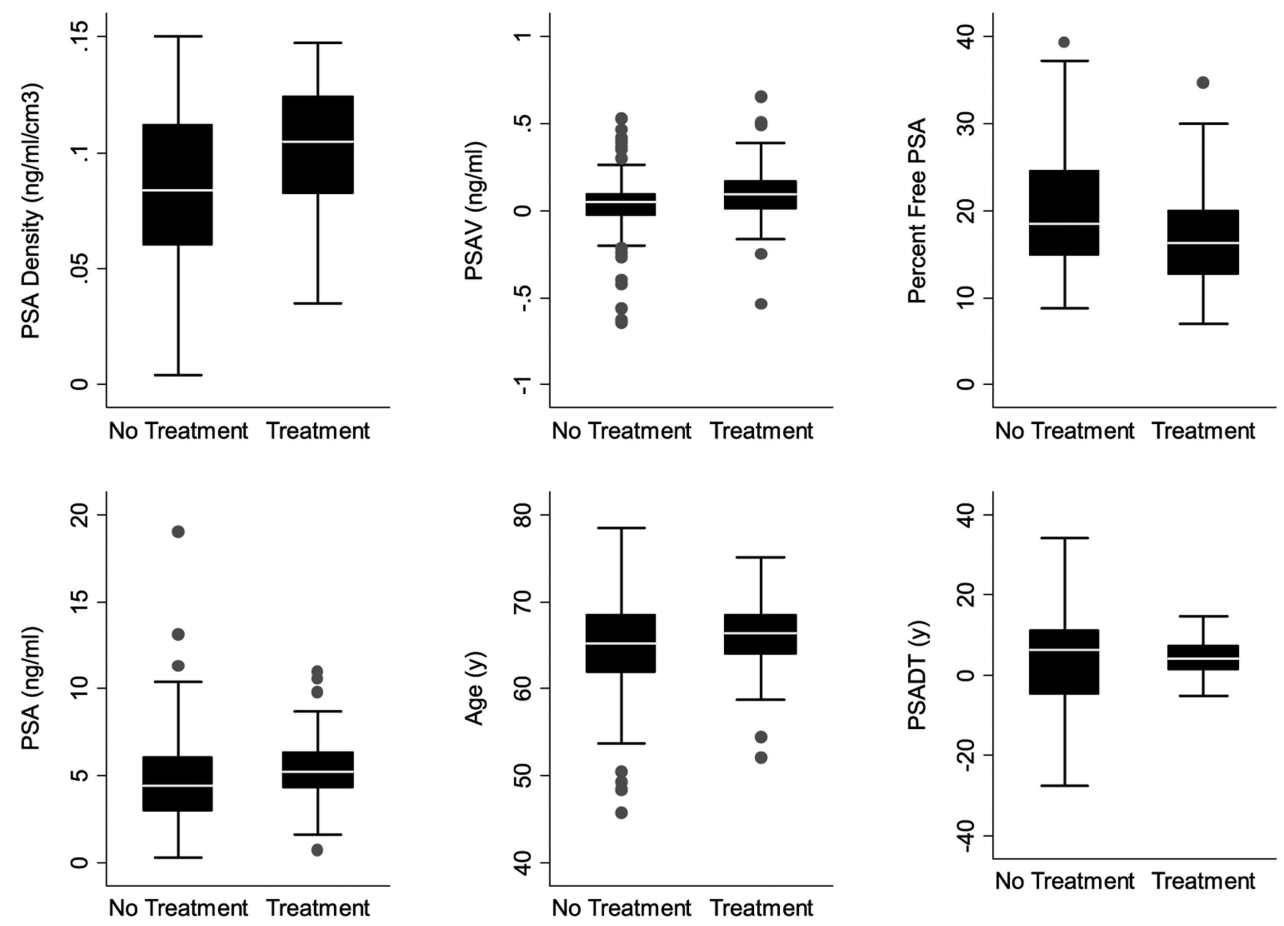

Fig. 2.

PSAD, PSAV, percent free PSA, PSA, age at diagnosis and PSADT in men who met all expectant management entry criteria, as described. Boxes represent IQR. Horizontal lines in boxes represent median. Vertical lines represent 1.5 times IQR with outlying PSADT values that exceeded 1.5 times IQR removed for illustration purposes. 


\section{Table 1}

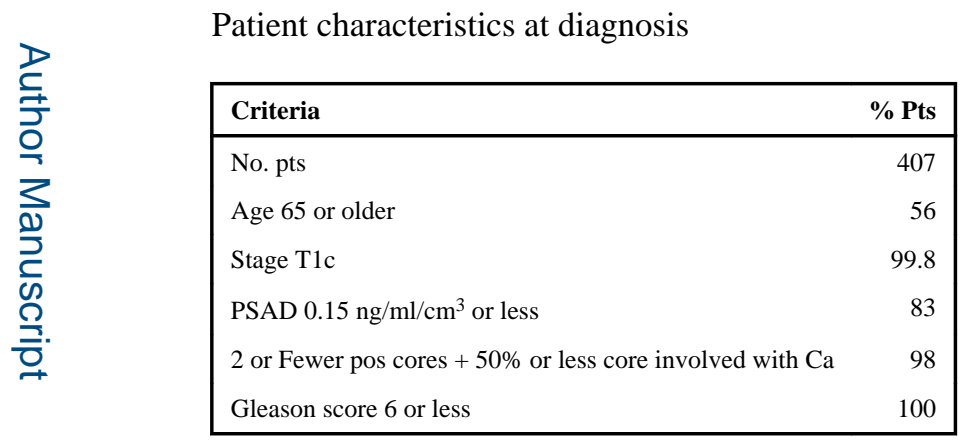

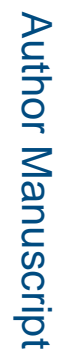

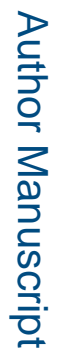

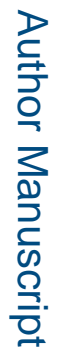

J Urol. Author manuscript; available in PMC 2015 April 08. 


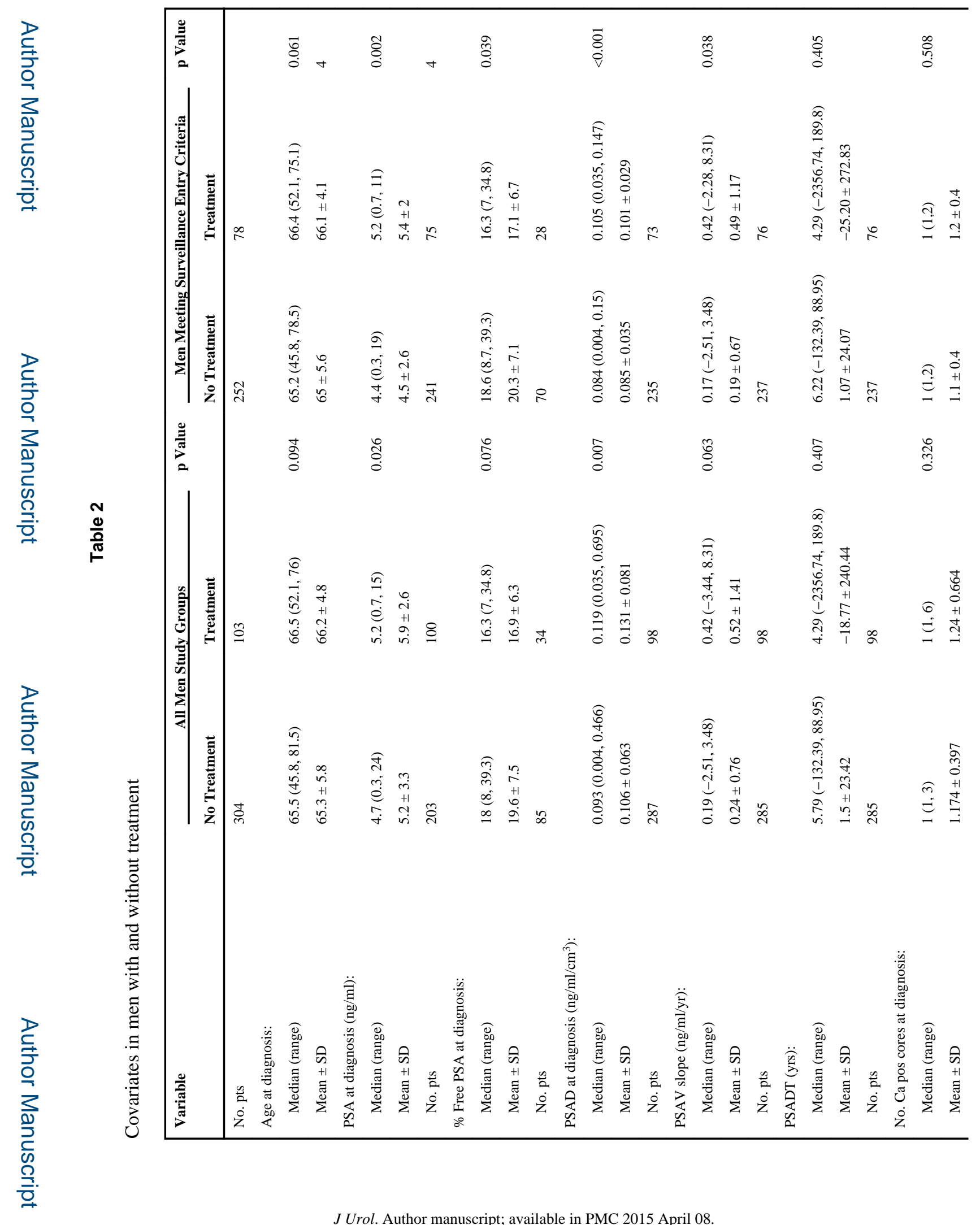




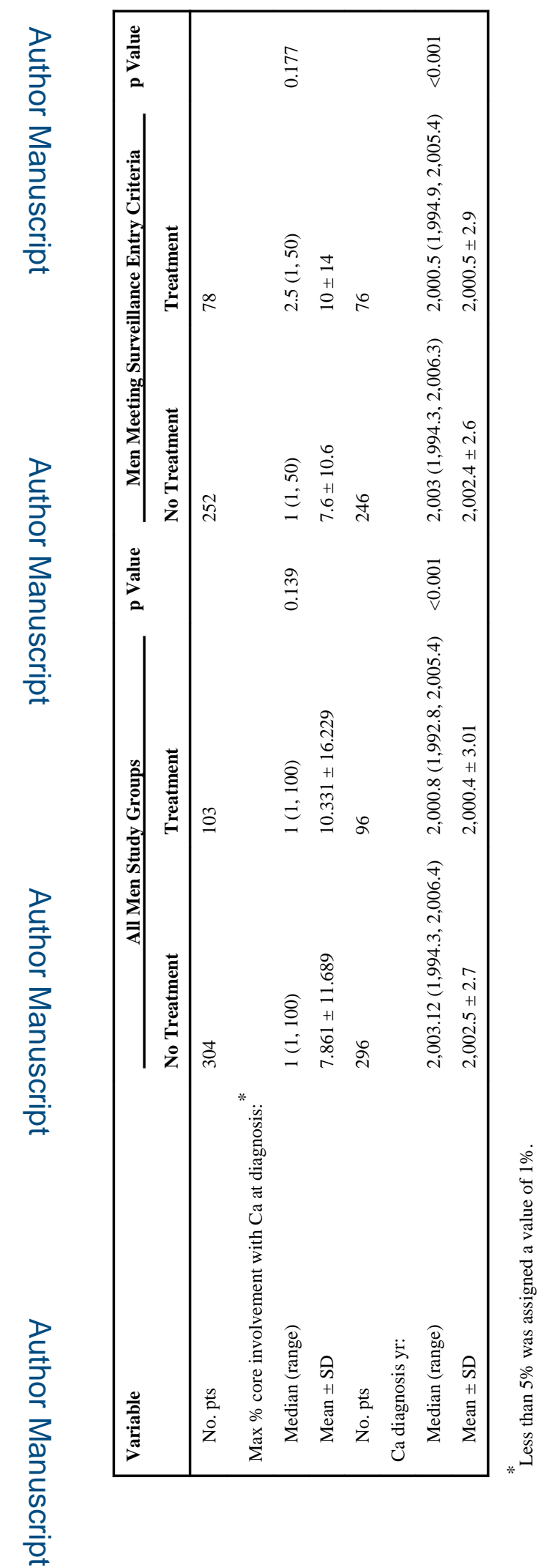

J Urol. Author manuscript; available in PMC 2015 April 08. 
Table 3

Cox regression analysis multivariate model for predicting delayed intervention in men treated expectantly

\begin{tabular}{|lllllll|}
\hline & \multicolumn{3}{c}{ All Men } & & \multicolumn{2}{c|}{ Men Meeting Entry Criteria ${ }^{\dagger}$} \\
\cline { 2 - 3 } Variable & Adjusted RR (95\% CI) & $\mathbf{p}$ Value & & Adjusted RR (95\% CI) & p Value \\
\hline Age at diagnosis & $1.056(1.013,1.1)$ & 0.011 & & $1.078(1.022,1.137)$ & 0.006 \\
PSA at diagnosis & $0.972(0.893,1.059)$ & 0.52 & & $1.023(0.892,1.173)$ & 0.745 \\
PSA density at diagnosis/0.01 U change & $1.027(0.992,1.063)$ & 0.135 & & $1.109(1.011,1.217)$ & 0.029 \\
PSA slope & $1.207(0.991,1.469)$ & 0.061 & & $1.274(1.037,1.565)$ & 0.021 \\
No. Ca pos cores & $1.192(0.811,1.753)$ & 0.371 & & $1.182(0.631,2.212)$ & 0.602 \\
Max \% core involvement with Ca & $1.009(0.997,1.022)$ & 0.141 & $1.015(0.996,1.033)$ & 0.118 \\
Diagnosis yr & $0.881(0.816,0.951)$ & 0.001 & $0.874(0.799,0.956)$ & 0.003 \\
\hline
\end{tabular}

* Total of 351 patients, including 87 with delayed curative intervention and 264 untreated.

${ }^{\dagger}$ Total of 288 patients, including 70 with delayed curative intervention and 218 untreated.

${ }^{\ddagger}$ Regression of PSA on time using all PSA values after cancer diagnosis. 